\title{
Sustainable Lightweight Wireless Communication Stack
}

\author{
Hemant Jeevan Magadum*, Arun C S**, Dr. Ravikumar P. *** \\ *ITNS, CDAC, Thiruvananthapuram-695033 \\ **Shree Buddha College Of Engineering, Thiruvalla \\ ***ITNS, CDAC, Thiruvananthapuram-695033
}

\begin{abstract}
A light weight wireless communication stack based on wireless communication establishment. It handles data received over the air and send serially with security checking and acknowledgement. Also it transmits data over the air which is received serially with security checking and acknowledgement. It gives status of alive routers on coordinator's UART port.
\end{abstract}

Keywords: Wireless, Zigbee,

\section{INTRODUCTION}

Lightweight wireless communication is a low-cost, low-power, wireless mesh network standard. Zigbee is a high level communication protocol which is used to create low power personal area network. It is based on 802.15.4 specification. ZigBee operates in the industrial, scientific and medical (ISM) radio bands; $868 \mathrm{MHz}$ in Europe, 915 $\mathrm{MHz}$ in the USA and Australia, and $2.4 \mathrm{GHz}$. (Introduce ZigBee before talking about its operation) ZigBee incorporates a complex routing algorithm called Adhoc distance vector routing But in modified stack no routing algorithm exists. The zigbee stack consists of main 4 layers. These layers are Physical layer, MAC layer, Network layer and Application layer .

In the lightweight wireless communication stack application and network layer modified for UART and wireless communication. In the application layer, written code to handle data received over the air and transmit the data over the air. In the network layer beacon frames are formed and from the coordinator network layer will send beacons in every second.

\section{LITERATURE REVIEW}

Dr. Narmada Alaparthi [1] proposed adaptation layer to include above network layer of Zigbee stack. It addresses major issues like header overhead, limited bandwidth, address scheme and node energy and large memory requirement of TCP/IP. It uses low energy and memory.

Li Pengfei[2] proposed wireless technology development and more attention of uses of zigbee in industry automation, social communication and smart home. Through study verified that zigbee is low rate, low cost and low consumption wireless communication.

Priyanka Deshpande[3] described techniques for improving throughput of wireless sensor network.It explains basic concept of zigbee, wireless sensor network(WSN) and types of zigbee devices. When data transferred to one sensor node to another node, the throughput of sensor node decreases because of packet collision and high network traffic. This problem can overcome using different topology like distributed throughput optimization for zigbee cluster tree network, time shift grouping access in IEEE 802.15.5 MAC Beacon mode for Layered-Tree networks, Adaptive IEEE 802.15.4 MAC[4] for throughput \& energy optimization, CoZi: basic coding for better bandwidth utilization in zigbee sensor networks

\section{SUSTAINABLE LIGHTWEIGHT WIRELESS COMMUNICATION STACK}

Lightweight wireless communication protocol is intended for applications which requires low data rate and low power consumption. No routing algorithm exists in modified stack. lightweight wireless communication devices are of three types Coordinator, Router and End Device. End device used where data communication is not frequent . End device is having enough functionality to communicate with parent node either coordinator or router. In idle condition, end device goes to sleep mode which gives long battery life. In lightweight wireless communication stack, we are concentrating on communication between coordinator and router . It supports communication between 1 coordinator and 15 routers. The role of Coordiator and Router is given below. 
- Coordinator (ZC): Coordinator forms the root of the network tree and it bridge to other networks. There is exactly one lightweight wireless communication coordinator in each network since it is the device that started the network originally. It stores information about the network, including acting as the Trust Center \& repository for security keys.

- $\quad$ Router (ZR):A router can act as an intermediate router, passing on data from other devices. It can capable to communicate with coordinator for data transfer .

In lightweight wireless communication stack, the routers will respond to the beacons transmitted by the coordinator and this ensures the presence or absence of any device in the network. If any of the routers failed to respond to the beacons, this will indicate that the particular device is not there in the network and the coordinator will write this situation in the UART. An acknowledgement system in the application layer track the acknowledgements of the transmitted frames from the UART. Coordinator broadcasts the data; routers receive the data and sends acknowledgement for the received frame. A sequence number is appended with packets transmitted from UART. At the receiver side, packets with sequence no is written to UART. No sequence number for beacon packets and hence it will not be written to UART.

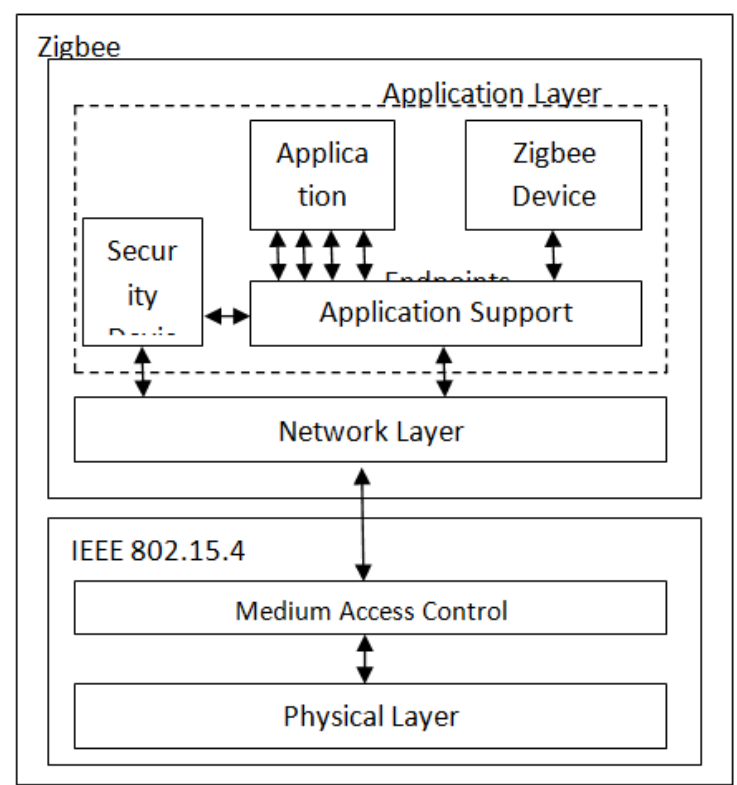

(Fig 1 - Zigbee stack Layers)

\section{COMMUNICATION STEPS}

The raw lightweight wireless communication stack form network automatically by sending match description request without any user interruption. The first step is to disable all the keys in the stack. Next step is to find when to send match description request. Communication will be possible only after match description request so it should be done before sending any data. When a lightweight wireless communication device is powered up it passes through different states like initialization state, orphan state, network manager/ lightweight wireless communication coordinator, router or as an end device, depends on the compiler settings.

An ideal state to send match description request is when a device changes from initialization state to orphan state or from orphan state to router state. The transition from orphan state to router state is most suitable for sending match description request. When a transition from orphan state to router state is detected, ZDO_STATE_CHANGE event is triggered. Match description request is sent when ZDO_STATE_CHANGE event is triggered. Match description request is sent from every router to coordinator. Now the device is ready for communication.

\section{ACKNOWLEDGE MECHANISM}

It will be a great advantage if the transmitter knows whether the data packet is delivered to the correct destination or not. A data or MAC command frame shall be sent with the acknowledgment request subfield of its frame control field set appropriately for the frame. A beacon or acknowledgment frame shall always be sent with the acknowledgment request subfield set to 0 . Similarly, any frame that is broadcast shall be sent with its acknowledgment request subfield set to 0 .

A frame transmitted with the acknowledgment request subfield of its frame control field set to 1 shall be acknowledged by the recipient. If the intended recipient correctly receives the frame, it shall generate and send an acknowledgment frame containing the same data sequence number from the data or MAC command frame that is being acknowledged. The transmission of an acknowledgment frame in a non-beacon enabled PAN or in the CFP shall commence a Turnaround Time symbols after the reception of the last symbol of the data or MAC command frame. The transmission of an acknowledgment frame in the CAP shall commence at a back off slot boundary. In this case, the transmission of an acknowledgment frame shall commence between aTurnaroundTime and (aTurnaroundTime + aUnitBackoffPeriod) symbols after the reception of the last symbol of the data or MAC command frame. Fig. 2 shows how a single frame of data transmitted from an originator to a recipient with an acknowledgment. In this case, the originator indicates to the recipient that it requires an 
acknowledgment by transmitting the data frame with the acknowledgement request (AR) subfield set to 1 .

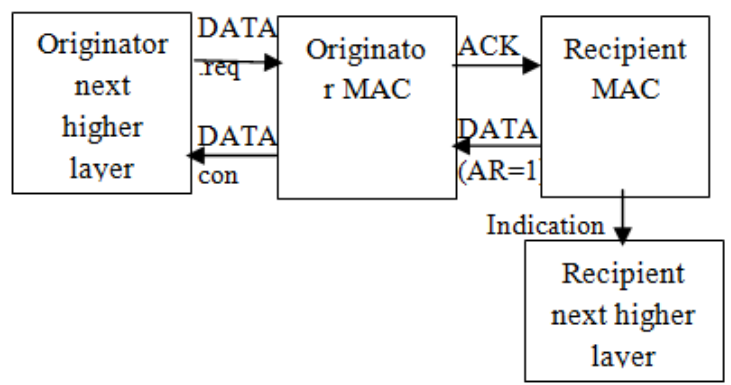

(Fig 2. Data transmission with acknowledgement)

The communication scheme adopted in such a way that the coordinator broadcasts all message packets and the routers sent messages to router. Since the router has a fixed address of $0 \times 0000$, the transmission from router to coordinator is unicast. But according to lightweight wireless communication protocol their will not be an acknowledgement for the broadcasted frames. That is, the packets broadcasted from coordinator to router will not be acknowledged. So from the coordinator side it is impossible to know the status of transmitted packets with lightweight wireless communication.

An acknowledgement system should be implemented in the application level to ensure that the transmitted packets are successfully received at the receiver and the packets are processed. So an acknowledgement packet is formed in the router. The acknowledgment packet from the router is entirely different from the default lightweight wireless communication MAC level acknowledgement.

The application level acknowledgement packet consists of a CLUSTER ID, ROUTER ID, sequence number and the status of transmission. At the coordinator side, after receiving the acknowledgement packet, ROUTER ID is written to UART. Thus the user is informed of the status of the transmitted packet.

Fig3 shows the structure of the acknowledgment frame, which originates from the MAC sublayer. The MAC acknowledgment frame is constructed from an MHR and an MFR. The MHR contains the MAC frame control and data sequence number fields. The MFR is composed of a 16 bit FCS. The MHR and MFR together form the MAC acknowledgment frame (i.e., MPDU). The MPDU is passed to the PHY as the PHY acknowledgment frame payload, (i.e., PSDU). The PSDU is prefixed with the SHR, containing the preamble sequence and SFD fields, and the PHR containing the length of the PSDU in octets. The SHR, PHR, and PSDU together form the PHY acknowledgment packet, (i.e., PPDU)

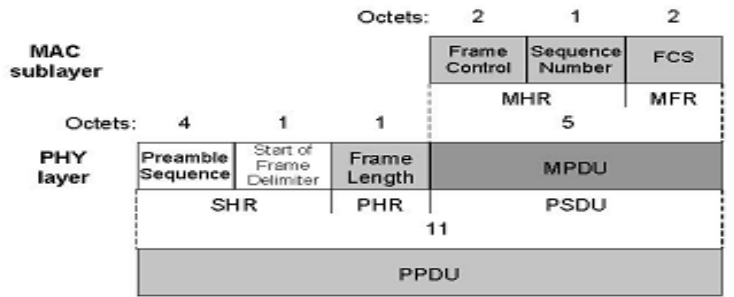

Fig3: structure of the acknowledgment frame

\section{MAC COMMAND FRAME}

Fig.4 shows the structure of the MAC command frame, which originates from the MAC sub layer. The MSDU contains the command type field and command specific data, called the command payload. The MSDU is prefixed with an MHR and appended with an MFR. The MHR contains the MAC frame control, data sequence number, and addressing information fields. The MFR contains a 16 bit FCS. The MHR, MSDU, and MFR together form the MAC command frame.

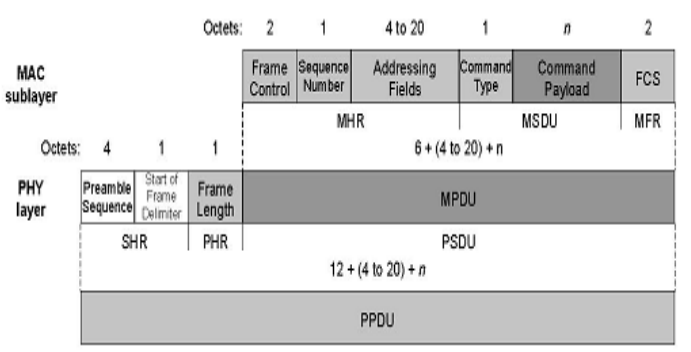

(Fig4: MAC command frame)

The MPDU is then passed to the PHY as the PHY command frame payload, (i.e., PSDU). The PSDU is pre-fixed with an SHR, containing the preamble sequence and SFD fields \& a PHR containing the length of the PSDU in octets. The preamble sequence enables the receiver to achieve symbol synchronization. The SHR, PHR, and PSDU together form the PHY command packet.

\section{BEACON IMPLEMENTATION}

Lightweight wireless communication supports 2 types of network namely beacon enabled network and non beacon network. Our application needs beacon synchronized network but IT stack is supports only non beacon network. At the beginning i.e., during network formation time routers send beacons to indicate their presence to coordinator. After that no beacon packets are sending. These beacon packets are sent from the MAC layer and the developer has no control over it. They are meant to form the network formation.

Fig.5 shows the structure of the beacon frame, which originates from the MAC sub layer. A coordinator can transmit network beacons in a beacon-enabled network. The MAC service data unit 

ISSN : 2248-9622, Vol. 7, Issue 5, ( Part -3) May 2017, pp.65-68

(MSDU) contains the super frame specification, pending address specification, address list, and beacon payload fields. The MSDU is prefixed with a MAC header (MHR) and appended with MAC footer (MFR). The MHR contains the MAC frame control fields, beacon sequence number (BSN), and addressing information fields. The MFR contains a 16 bit frame check sequence (FCS). The MHR, MSDU, and MFR together form the MAC beacon.

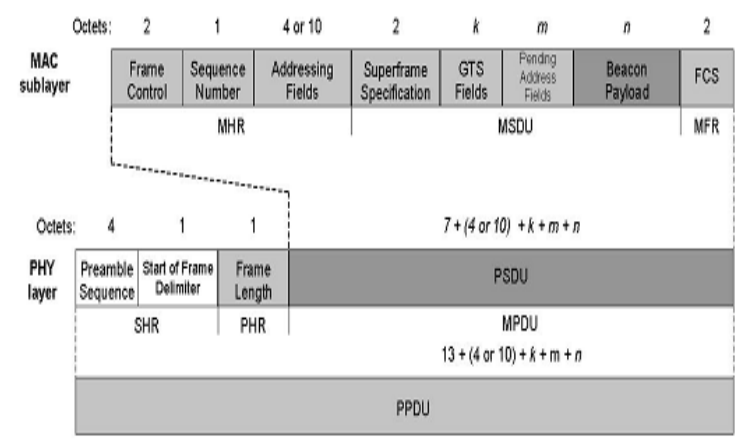

Fig5. Beacon frame structure

The data payload is passed to the MAC sub layer and is referred to as the MSDU. The MSDU is prefixed with an MHR and appended with an MFR. The MHR contains the frame control, sequence number, and addressing information fields. The MFR is composed of a 16 bit FCS. The MHR, MSDU, and MFR together form the MAC data frame, (i.e., MPDU). The MPDU is passed to the PHY as the PHY data frame payload, (i.e., PSDU). The PSDU is prefixed with an SHR, containing the preamble sequence and SFD fields, and a PHR containing the length of the PSDU in octets. The preamble sequence and the data SFD enable the receiver to achieve symbol synchronization. The SHR, PHR, and PSDU together form the PHY data packet.

In our application, it is desired to know the status of other routers from the coordinator. For this requirement a network layer beacon is implemented. This beacon is sent from the network layer of coordinator every second and other and the routers will send the response packet to the coordinator. From the serial response packet the user can identify the status of the network and can perform any desired action. Provision present to identify these 16 routers.

Beacon packet broadcasted from the coordinator mainly contains a header, and 2 bytes of data field. Each bit in the data field corresponds to a particular slave. Only the first bit in the data field is one. The first router will make no shift to the data, the second router will make 1 shift to the router, the third router will make 3 shifts it the router and so on. The routers will send the shifted data to the coordinator. By examining the data field in the response packet, coordinator knows which slave has failed and can perform the desired action.

\section{CONCLUSION}

Coordinator forms available routers network automatically. Network layer beacon is implemented and transmitted from coordinator every second. The total number of routers configurable at coordinator side and status of alive routers available as per beacon acknowledgement. Ensured range of wireless communication is more than 150 meter and transmitted power is $4.5 \mathrm{dBm}$. Coordinator and router tested by interfacing with Wireless Traffic Control System (WiTraC). It reduces uart transmission overhead from master to wireless modules because of wireless module having inbuilt every second transmission instead of WiTraC master controller. Future work is fine tuning lightweight swireless communication stack.

\section{ACKNOWLEDGEMENTS}

I wish to acknowledge the following colleagues from CDAC who provided guidance.

\section{REFERENCES}

[1]. Dr. Narmada Alapathi and Dr. P. Sudhakara Rao, "Adaptation layer towards integration of Zigbee and IP stack", published in IEEE International Conference on Control and Dynamic Optimization techniques(ICACDOT) I2IT Pune 2016.

[2]. Li Pengfei, Li Jiakun, Nei Luhua, Wang Bo, "Research and application of Zigbee Protocol Stack", published in IEEE International Conference on measuring technology an d Mechatronics Automation 2010.

[3]. Priyanka Deshpande and Mangala Madankar, "Techniques improving throughput of wireless sensor network:A survey", published in IEEE International Conference on circuit, power and computing Technologies[ICCPCT] 2015IEEE 802.15.4 Specification.

[4]. IEEE 802.15.4 Specification.

[5]. Gutierrez: Low-Rate Wireless Personal Area Networks, IEEE Press,2007.

[6]. "ZigBee Architecture Overview", Oslo, Norway June 2005.

[7]. CC2520 and CC2530 datasheet.

[8]. IEEE 802.15.4: Wireless Medium Access Control (MAC) and Physical Layer(PHY) specifications for Low-Rate Wireless Personal Area Networks (WPANs), Sept,2006. 